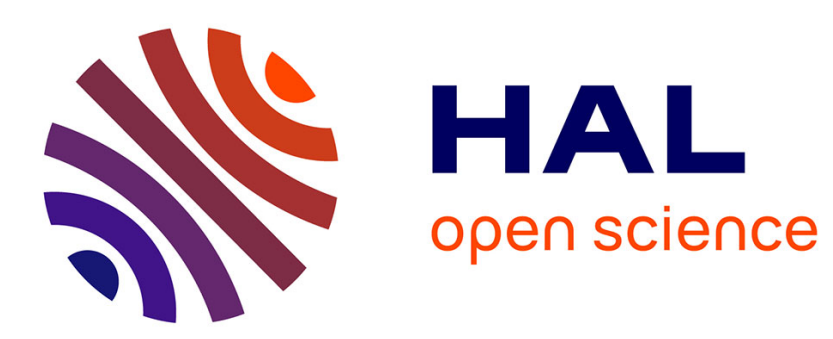

\title{
Understanding Collateral Evolution in Linux Device Drivers
}

\author{
Yoann Padioleau, Julia L. Lawall, Gilles Muller
}

\section{To cite this version:}

Yoann Padioleau, Julia L. Lawall, Gilles Muller. Understanding Collateral Evolution in Linux Device Drivers. [Research Report] RR-5769, INRIA. 2005, pp.18. inria-00070251

\section{HAL Id: inria-00070251 \\ https://hal.inria.fr/inria-00070251}

Submitted on 19 May 2006

HAL is a multi-disciplinary open access archive for the deposit and dissemination of scientific research documents, whether they are published or not. The documents may come from teaching and research institutions in France or abroad, or from public or private research centers.
L'archive ouverte pluridisciplinaire HAL, est destinée au dépôt et à la diffusion de documents scientifiques de niveau recherche, publiés ou non, émanant des établissements d'enseignement et de recherche français ou étrangers, des laboratoires publics ou privés. 
INSTITUT NATIONAL DE RECHERCHE EN INFORMATIQUE ET EN AUTOMATIQUE

\section{Understanding Collateral Evolution in Linux Device Drivers}

Yoann Padioleau — Julia L. Lawall — Gilles Muller

$$
\mathbf{N}^{\circ} 5769
$$

Novembre 2005

Thème COM

apport

de recherche 



\title{
Understanding Collateral Evolution in Linux Device Drivers
}

\author{
Yoann Padioleau, Julia L. Lawall, Gilles Muller \\ Thème COM - Systèmes communicants \\ Projet Obasco \\ Rapport de recherche $\mathrm{n}^{\circ} 5769$ - Novembre 2005 - 18 pages
}

\begin{abstract}
In a modern operating system (OS), device drivers can make up over $70 \%$ of the source code. Driver code is also heavily dependent on the rest of the OS, for functions and data structure defined in the kernel and driver support libraries. These two properties together pose a significant problem for OS evolution, as any changes in the interfaces exported by the kernel and driver support libraries can trigger a large number of adjustments in dependent drivers. These adjustments, which we refer to as collateral evolutions, may be complex, entailing substantial code reorganizations. Collateral evolution of device drivers is thus time consuming and error prone.

In this paper, we present a qualitative and quantitative assessment of the collateral evolution problem in Linux device driver code. We provide a taxonomy of evolutions and collateral evolutions, and show that from one version of Linux to the next, collateral evolutions can account for up to $35 \%$ of the lines modified in such code. We then identify some of the challenges that must be met in the future to automate the collateral evolution process.
\end{abstract}

Key-words: operating system, device driver, program transformation, software reengineering, software maintenance. 


\section{Comprendre les Évolutions Collatérales dans les Pilotes de Périphériques sous Linux}

Résumé : Dans un système d'exploitation moderne (OS), les pilotes de périphériques peuvent constituer jusqu'à $70 \%$ du code source. De plus, le code des pilotes de périphériques est hautement dépendant du reste de l'OS, pour des fonctions et structures de données définies dans le noyau et dans des bibliothèques de support aux pilotes. Ces deux propriétés ensemble posent un problème significatif pour l'évolution des OS. En effet, le moindre changement dans l'interface exportée par le noyau ou les bibliothèques de support peut déclencher un grand nombre d'ajustements dans le code des pilotes dépendants de ces interfaces. Ces ajustements, que l'on nomme des évolutions collatérales, peuvent être complexes, entraînant des réorganisations substantielles du code. Ainsi, les évolutions collatérales des pilotes de périphériques prennent à la fois beaucoup de temps et sont soumises à de nombreuses erreurs.

Dans ce papier, nous présentons une étude qualitative et quantitative du problème des évolutions dans les pilotes de périphériques sous Linux. Nous fournissons une taxinomie des évolutions et des évolutions collatérales, et nous montrons que d'une version à l'autre de Linux, les évolutions collatérales peuvent représenter jusqu'à $35 \%$ des lignes modifiées. Nous identifions ensuite quelques un des défis à résoudre dans le futur pour automatiser le processus d'évolution collatérale.

Mots-clés : système d'exploitation, pilote de périphérique, transformation de programme, génie logiciel, maintenance de programme. 


\section{Contents}

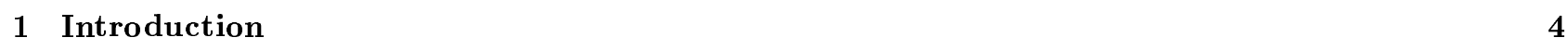

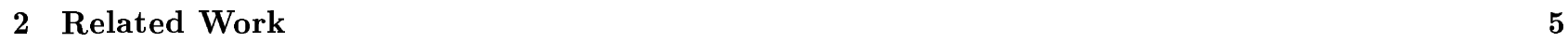

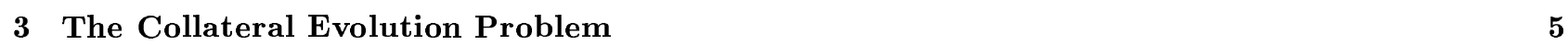

3.1 Linux support for devices . . . . . . . . . . . . . . . . . . . . . . 6

3.2 Taxonomv of interface changes and collateral evolutions that affect device-specific code . . . . . 6

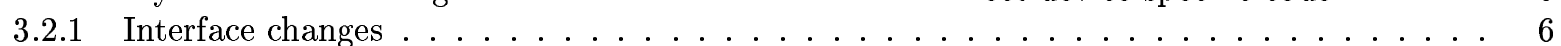

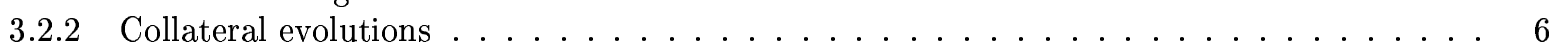

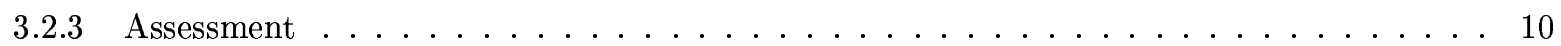

$\begin{array}{lll}4 \text { Case Studies } & 10\end{array}$

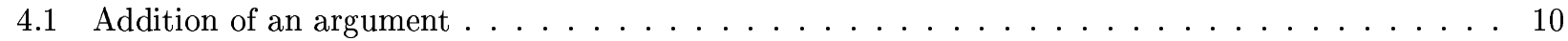

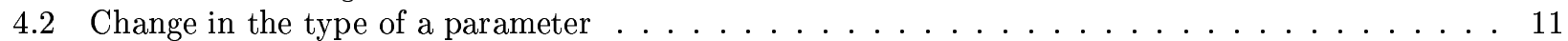

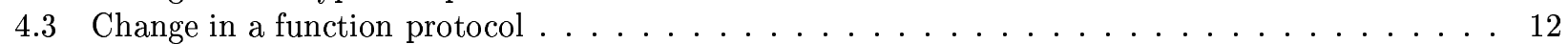

5 Quantitative Assessment 12

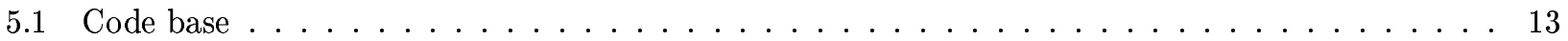

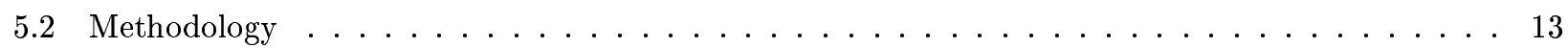

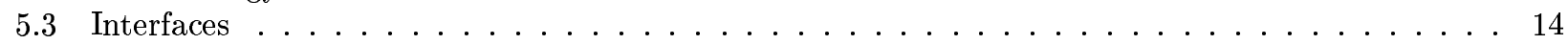

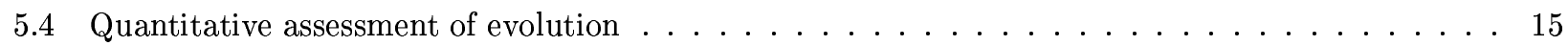

5.5 Quantitative assessment of collateral evolution $\ldots \ldots \ldots \ldots \ldots \ldots \ldots$

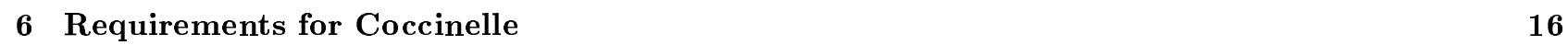

6.1 Support for $\mathrm{C}$ code $\ldots \ldots \ldots \ldots \ldots \ldots \ldots \ldots \ldots \ldots \ldots$

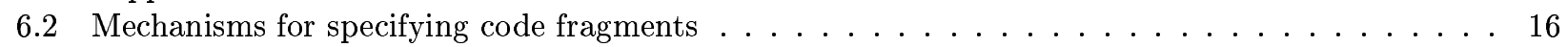

6.3 Mechanisms for describing relationships between code fragments $\ldots \ldots \ldots \ldots$

\begin{tabular}{lll}
\hline 7 & Conclusion & 17
\end{tabular} 
"Greg Kroah-Hartman has gotten [Linux] 2.6.13 off to a good start with a massive set of driver core patches. There are a fair number of API changes that come with this patch set, so the whole thing is worth a look. In-tree code has been fixed to use the new API, but, as always, maintainers of external code are on their own." http://1wn.net/Articles/140002/, June 23, 2005.

\section{Introduction}

One of the biggest problems in operating system (OS) development today is keeping device drivers up to date with evolutions in the rest of the OS. Device driver code can make up over $70 \%$ of a modern OS [4] and is heavily dependent on the rest of the OS for functions and data structures defined in the kernel and driver support libraries. Accordingly, any changes in the interfaces of the kernel or driver support libraries are likely to affect driver code, and restoring their correct behavior often entails modifications at many code sites. These modifications, which we refer to as collateral evolutions, may involve substantial code reorganizations. In practice, evolution may be hindered because the required collateral evolution of device drivers is both time-consuming and error-prone.

We examine the issues raised by collateral evolution in the context of Linux. Linux is currently undergoing rapid evolution, with even the so-called stable version 2.6 introducing more and more interface changes [18]. Furthermore, the size of the Linux driver code has more than doubled in the last five years. These factors suggest that collateral evolutions are increasingly becoming necessary in Linux. Indeed, a single collateral evolution may affect hundreds of code sites spread across many different files.

The problem of collateral evolution in Linux is further complicated by the difficulty of communicating precise information about the required modifications to driver maintainers. As Linux is an open source OS, many kinds of programmers contribute to its development. Indeed, driver maintainers are often not kernel experts, but instead experts in a given device or even ordinary users who find that their hardware is not adequately supported. Because the developers who update the kernel and driver support libraries often do not share a common language and expertise with device maintainers, documentation about complex collateral evolutions, if any, is often incomplete. As a result, we have observed that an evolution and dependent collateral evolutions may take several years to complete and may introduce bugs into previously mature code.

\section{This paper}

While substantial attention has been paid to how to design an OS, there has been little consideration of its subsequent evolution. In the case of device driver collateral evolution, the magnitude and complexity of the problem call for automated assistance. Accordingly, we plan to develop a tool, Coccinelle 1 that provides a formal notation for describing collateral evolutions and a transformation engine to assist developers in applying them.

General-purpose transformation systems have, however, often been found to be too high-level or too lowlevel for convenient use in practice. To produce a tool that is practical for use by OS developers and maintainers, the abstractions and transformations provided by Coccinelle should thus be targeted to the specific requirements of collateral evolution. In this paper, we analyze the range and scope of the collateral evolution problem to identify these requirements. Overall, we make the following contributions:

- We clarify the structure of Linux as it relates to collateral evolution, focusing attention on the interfaces of the kernel and driver support libraries, and provide a taxonomy of the main evolutions that occur in these interfaces.

- We identify a variety of collateral evolutions that evolutions in this taxonomy can entail, and present three examples out of the 45 that we have studied in detail. These examples illustrate both the complexity of performing collateral evolution and the bugs that can be introduced in mature code.

- Based on the identification of interface elements as potential triggers of collateral evolutions, we show that the likelihood of collateral evolutions is increasing, as not only the driver code size but also the complexity of the driver interfaces has doubled in the last five years.

- We have designed a tool that analyzes patch files to identify evolutions and collateral evolutions in Linux device-specific code. This tool gives a more accurate picture than the study of header files, as header files only indicate type changes and do not clearly distinguish what is to be used by the device-specific code from facilities that are shared by the kernel or driver support library implementation.

- Using our tool, we measure the number of evolutions affecting driver code in versions of Linux from 2.2 to 2.6 , including both type changes and

\footnotetext{
${ }^{1} \mathrm{~A}$ coccinelle is a ladybug, which is an insect that eats smaller bugs.
} 
changes involving argument values and usage protocols. We find that the number of these evolutions has been steadily increasing, with the socalled stable Linux 2.6 showing almost as many evolutions as its unstable predecessor, Linux 2.5.

- We then give an estimate of the work required for collateral evolution, based on the amount of modification required in driver files. Using our tool, we find that a significant portion (up to $35 \%$ ) of lines modified in Linux device-specific code from one version to the next are due to collateral evolutions. This result is particularly noteworthy because collateral evolutions serve only to maintain the current behavior, rather than improve it.

- Based on this study of collateral evolution in Linux, we identify some of the challenges that Coccinelle must meet.

The rest of this paper is organized as follows. Section 2 describes related work. Section 3 provides an assessment of the kinds of changes that occur in interfaces that affect device drivers and the collateral evolutions that these changes entail. Section 4 describes some of these collateral evolutions in detail. Section [5] quantifies various aspects of Linux evolution and collateral evolution. Section 6 proposes some features that a tool must provide to assist developers with collateral evolution. Finally, Section 7 presents some conclusions and ideas for future work.

\section{Related Work}

The work presented in this paper is directed toward automating the problem of updating driver code to conform to new interfaces. Previous work has suggested to address this problem using wrapper functions or virtual machines, thus leaving the driver code unchanged. Using these approaches, however, driver code does not benefit from improvements in the overall software architecture of the OS that could ease its future maintenance, e.g. to address new device requirements. The wrapper approach is furthermore not always sufficient, as some collateral evolutions such as the updating of calls to usb_submit_urb described in Section 4.1, depend on information that is only apparent in the driver code. In such cases, the chaos of coding styles induced by the wrapper approach makes the collateral evolution all the more difficult. Finally, we have observed the introduction and subsequent removal of wrapper functions in Linux code, suggesting that the Linux development community does not see them as a viable solution.

Recent years have seen a surge of interest in automatic detection of bugs in large pieces of software, including Linux [7, 8, 11, 17] and Windows [3]. These approaches rely on a collection of required kernel API usage patterns and detect code fragments that are inconsistent with these patterns. Nevertheless, an incorrectly done or overlooked collateral evolution may satisfy expected patterns without actually correctly restoring the behavior of the device driver. Detection of the error would require combining information about the original implementation of the driver with analysis of the new version.

Some attention has also been paid to OS evolution, including the evolution of drivers. Hassan has developed a tool that automatically extracts evolution information from versioning repositories and has been applied to OSes such as FreeBSD [13]. This approach, however, only associates each change to the enclosing function, and does not infer the kinds of relationships between changes needed to perform the collateral evolution automatically.

Evolutions and collateral evolutions are related to refactorings, a collection of fixed general-purpose transformations that reorganize the structure of a program without changing its semantics [12]. Refactorings, however, apply to the whole program, requiring accesses to all usage sites of affected definitions. In the case of Linux, however, the entire code base is not available, as many device drivers are developed outside the Linux source tree. There is currently no way of expressing or generating the effect of a refactoring on such external code.

Finally, if Linux were structured using components, as done in OSes such as Think [9, OSKIT [10] and K42 [2], it would be easier to identify its interfaces and detect their evolution.

\section{The Collateral Evolution Prob- lem}

Collateral evolution is required when evolutions in the kernel and driver support libraries induce changes in the interface with device-specific code. To characterize the collateral evolution problem, we first examine the structure of Linux support for devices and identify the kinds of changes that can occur in its interfaces. We then consider the range of collateral evolutions that these changes can entail.

In the following, we avoid the term "device driver," which can be interpreted either as including only the code that interacts directly with the device or as additionally including the relevant support libraries. Instead, we refer to the former as device-specific code and the latter as driver support libraries. Furthermore, we consider the kernel to be a driver support library except where specified otherwise. 


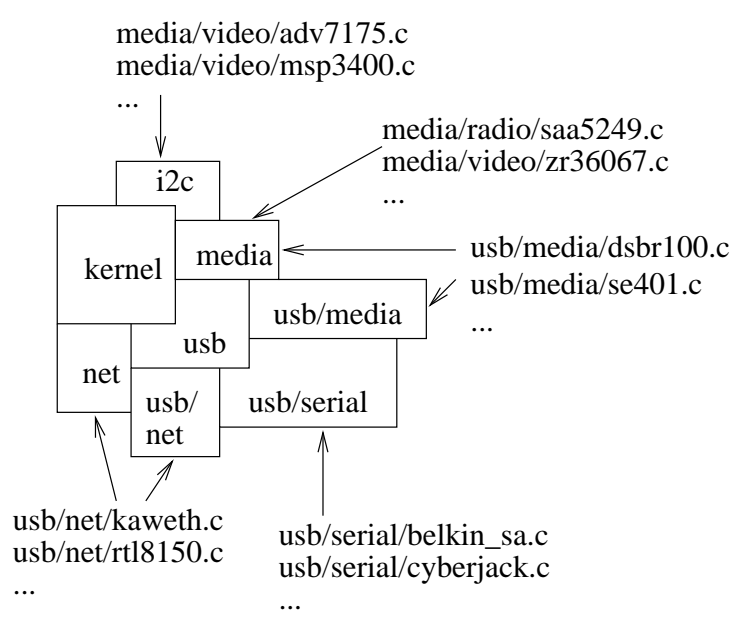

Figure 1: Hierarchical organization of services and associated dependencies

\subsection{Linux support for devices}

Linux support for devices is provided by a combination of generic services that are provided by the kernel, services generic to a device family that are provided by the driver support libraries, and services specific to a device that are provided by the device-specific code. As illustrated in Figure 1 these services are organized hierarchically, with all services depending on the kernel, specialized driver support libraries such as USB-serial depending on generic ones such as USB, and devicespecific files such as rt18150.c depending on one or more driver support libraries. Typically, these hierarchical relationships are reflected in the Linux directory structure. Nevertheless, as illustrated in Figure 1 by rt18150.c and others, cross-directory references are also possible.

The various driver support libraries (including the kernel) communicate with the device-specific code via interfaces. As Linux interfaces (i.e., header files) are insufficiently descriptive, we infer these interfaces from the external references made by the device-specific code. The driver support libraries and the devicespecific code mainly interact via function calls, both generic functions exported by the driver support libraries and device-specific callback functions provided by the device-specific code. A driver support library furthermore typically uses data structures to maintain the state of each relevant device. The types of these structures are defined in the interface and are instantiated by the device-specific code. These structures are then exchanged between the driver support library and the device-specific code on the invocation of the various interface functions. Finally, the interface of a driver support library includes a protocol for using the exported functions and data types. This protocol can specify features such as function-call sequencing and error-handling requirements.

Figure2 2 shows extracts of the rt18150 device-specific code, which has a typical structure. This code depends on the USB and network device support libraries. The initialization function, usb_rt18150_init (lines 4649), registers the device with the USB support library, providing it with a structure, rt18150_driver (lines 1-5), that contains a number of callback functions required by the USB support library. One of these functions is rt18150_probe (lines 31-44), which when invoked by the USB library registers the device with the network device support library in a similar manner. Figure2]also shows the function rt18150_start_xmit (lines 7-29), which is among the callback functions imported by the network device support library. This function illustrates the use of a variety of functions exported by both libraries, the exchange of data structures, and the instantiation of protocols. The relevant extracts of the USB and network device interfaces are shown in Figure 3

\subsection{Taxonomy of interface changes and collateral evolutions that affect device-specific code}

When an evolution in a driver support library affects its interface, collateral evolutions must be made in all dependent device-specific files. For example, when a library function $f$ gains a new argument, device-specific code that uses $f$ must be modified to construct an appropriate argument value. In this section, we first provide a taxonomy of changes that are possible in driver support library interfaces and then consider the range of collateral evolutions that these changes can entail.

\subsubsection{Interface changes}

As motivated in Section 3.1, the interface of a driver support library includes exported functions, imported callback functions, data structures, and protocols. Evolutions in the driver support library can have arbitrary effects on one or more of these elements. Figure 4 provides a taxonomy of these effects, obtained by considering systematically the information included in each case and the changes that can occur in this information. In a study of Linux 2.5, described below, we have observed all of these changes in driver support library interfaces. We thus expect this taxonomy to apply to other Linux versions as well.

\subsubsection{Collateral evolutions}

A collateral evolution represents a side effect on the context in which an interface element is used. While interface elements themselves are intrinsically restricted and fixed, their usage context consists of arbitrary 


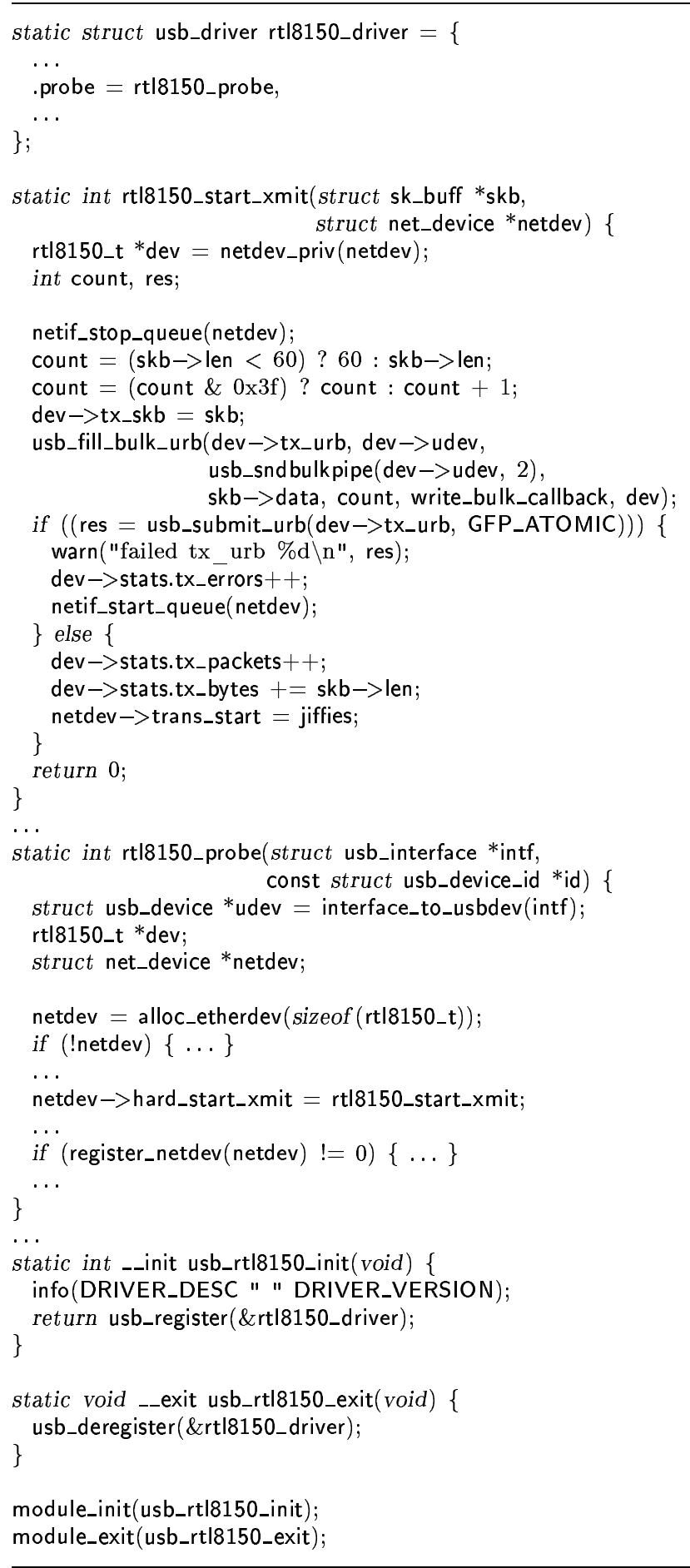

Figure 2: Extracts of the rt18150.c in Linux 2.6.13

\begin{tabular}{l|l|l|}
\cline { 2 - 3 } 1 & USB interface \\
\cline { 2 - 3 } 2 & Exported functions & usb_fill_bulk_urb, \\
3 & & usb_sndbulkpipe, \\
5 & & usb_submit_urb, \\
6 & & interface_to_usbdev \\
7 & & usb_register,usb_deregister \\
\cline { 3 - 3 } 8 & Imported callback & none \\
9 & functions & \\
\cline { 2 - 3 } 10 & Data structures & rtl8150_driver, dev->udev of type \\
11 & & struct usb_device \\
\cline { 2 - 3 } 13 & Protocol & usb_fill_bulk_urb if used precedes \\
14 & & usb_submit_urb \\
\hline
\end{tabular}

\begin{tabular}{l|l|l|}
15 & \multicolumn{2}{|l|}{ Network device interface } \\
\cline { 2 - 3 } 17 & Exported functions & netif_stop_queue, \\
18 & & netif_start_queue, alloc_etherdev \\
19 & & register_netdev \\
20 & Imported callback & rt18150_start_xmit \\
21 & functions & \\
22 & Data structures & netdev of type struct net_device \\
23 & Protocol & netif_start_queue follows \\
24 & & netif_stop_queue on error. \\
25 & &
\end{tabular}

27 Figure 3: Extracts of the USB and network device in28 terfaces

29

30

31

32

34

35

36
37

38

39

40
41

\begin{tabular}{|c|c|}
\hline 2 Exported functions & $\begin{array}{l}\text { - add/drop arguments } \\
\text { - change function name } \\
\text { - change return type }\end{array}$ \\
\hline $\begin{array}{l}\text { Imported callback } \\
\text { functions }\end{array}$ & $\begin{array}{l}\text { - add/drop required parameters } \\
\text { - change required return type }\end{array}$ \\
\hline Data structures & $\begin{array}{l}\text { - split or merge structures } \\
\text { - introduce layers of indirection } \\
\text { - convert a structure field reference } \\
\text { to a getter/setter function call }\end{array}$ \\
\hline Protocols & $\begin{array}{l}\text { - add or drop required calls } \\
\text { - change sequencing } \\
\text { - change locking requirements } \\
\text { - add required error checking }\end{array}$ \\
\hline
\end{tabular}

Figure 4: Changes that can occur in a driver support library interface 
code, and can vary widely from one device-specific file to another. It is thus not possible to develop an exhaustive taxonomy of all possible collateral evolutions. Instead, we have made a careful study of 45 collateral evolutions in the various subversions of Linux 2.5, based on several hundred potential collateral evolutions that we have identified. The studied collateral evolutions affect over one thousand files. We furthermore believe that these examples are representitive of the range and scope of collateral evolution in Linux because Linux 2.5 is an unstable version, in which many evolutions and collateral evolutions occur, and because they cover all of the identified interface changes.

In the rest of this section, we provide an overview of the kinds of collateral evolutions that we have identified in our study. Representitive examples among the 45 collateral evolutions that we have studied are shown in Figure 5 The line numbers in the text below refer to the lines in this figure.

Library functions We first consider the collateral evolutions required in response to changes in the signature of a library function, including its arguments, name, and return type.

Adding an argument to a library function or changing the type of an existing argument requires constructing a new value. In simple cases, the new value is a constant (line 1), a variable already bound in the current function (line 3), or a fixed transformation of the current argument, such as adding a structure field reference (line 4). In many cases, however, the addition of a new argument or change in an argument type requires substantial code rewriting, possibly depending on control and data flow information or external knowledge. For example, when the type of the argument changes to a new structure type it is necessary to create and initialize a new structure value as well as modifying the function call (line 6).

Dropping an argument is generally straightforward, since the argument has no impact on the context (line 8). It may, however, be desirable to remove any code involved in computing the argument value.

Changing the name of a library function is straightforward if the change is performed uniformly. It is more difficult, however, if the choice of the new function depends on the context. This is best illustrated by the case of bus_to_virt and related functions (line 14). In Linux 2.5.4, these functions were replaced by a functions having names beginning with "isa_," apparently to force programmers to consider whether the functionality of bus_to_virt was appropriate for the given context [19]. The renaming of the function was, however, not performed uniformly across the driver source code, leading to a flood of complaints in the Linux mailing lists [15, 25, 26]. As a result, in Linux 2.5.8, wrapper functions were introduced defining bus_to_virt etc. to their "isa_" counterparts, thus nullifying the benefit of the evolution.

Function names also change when a collection of functions defined by the device-specific code are unified into a single library function (line 15). The collateral evolution requires both recognizing and eliminating the device-specific definitions, which may exhibit inessential syntactic variations, as well as updating the call sites.

Changes in function return types typically derive from changes in error handling. When the return type of a library function changes from void to a type such as int that indicates an error condition, device-specific code has to be modified to introduce appropriate error handling. Often the device-specific code responds to the error by itself returning prematurely with an error code. In this case, the collateral evolution must take care to release any locally allocated resources. In other cases, it is the semantics, not the type of the return value that changes. In early versions of Linux, 0 was often used to indicate an error and 1 to indicate success. Gradually, there has been a shift to the use of more informative error codes, such as -EIO and -ENODEV. When a library function adopts the more informative error reporting strategy, collateral evolution is required at the call sites to invert the sense of 0 and possibly to introduce specialized error handling depending on the kind of error that is indicated by the return value. Similar issues occur when a library-specific return type is introduced.

Device-specific callback functions We next consider the collateral evolutions required when the interface exported by a driver support library specifies changes in the signature of an imported device-specific callback function.

In some cases, a parameter is added to a devicespecific callback function to harmonize the interface with an instance of the function that needs the additional information (line 17). In such cases, no further collateral evolution is required. On the other hand, a new parameter may supersede information previously computed by the function (line 18). In that case, careful slicing is required to eliminate the computation of this value, while leaving the other computations on which the function definition still depends. Finally, a new parameter can be added because the function will directly or indirectly call a library function that needs this information as a new argument (line 19). In this case, the new parameter has to be transmitted to all intervening function calls.

Dropping a parameter or changing its type means that the original value must be reconstructed if it is needed by the function (line 20). This can require pervasive changes in the function definition. 
Library function definitions

\begin{tabular}{|c|c|c|c|}
\hline \multicolumn{4}{|c|}{ Add argument/change argument type } \\
\hline & Version & Function & New value \\
\hline 1 & 2.5 .53 & pnp_activate_dev & NULL \\
\hline 2 & 2.5 .22 & end_request & CURRENT \\
\hline 4 & 2.5 .16 & usb_stor_clear_halt & field of existing argument \\
\hline 5 & 2.5 .54 & dev_get(set)_drvdata & subexpression of existing argument \\
\hline 6 & 2.5 .59 & agp_(un)register_driver & newly created and initialized global structure \\
\hline \multicolumn{4}{|r|}{ Drop argument } \\
\hline & Version & Function & Context effect \\
\hline 8 & 2.5 .63 & pnp_activate_dev & none \\
\hline 9 & 2.5 .70 & acpi_hw_low_level_read(write) & none \\
\hline \multicolumn{4}{|r|}{ Change function name } \\
\hline & Version & Renamed function & Function selection strategy \\
\hline 14 & 2.5 .4 & $\begin{array}{l}\text { bus_to_virt, virt_to_bus, } \\
\text { and page_to_bus }\end{array}$ & context-dependent, does not follow directory structure \\
\hline 15 & 2.5 .50 & sched_event & function moved from device-specific code to driver support library \\
\hline \multicolumn{4}{|r|}{ Change return type } \\
\hline & Version & Function & Effect \\
\hline 16 & 2.5 .20 & acpi_hw_register_read, etc. & add/adjust error checking using the acpi_status type \\
\hline
\end{tabular}

Driver function definitions

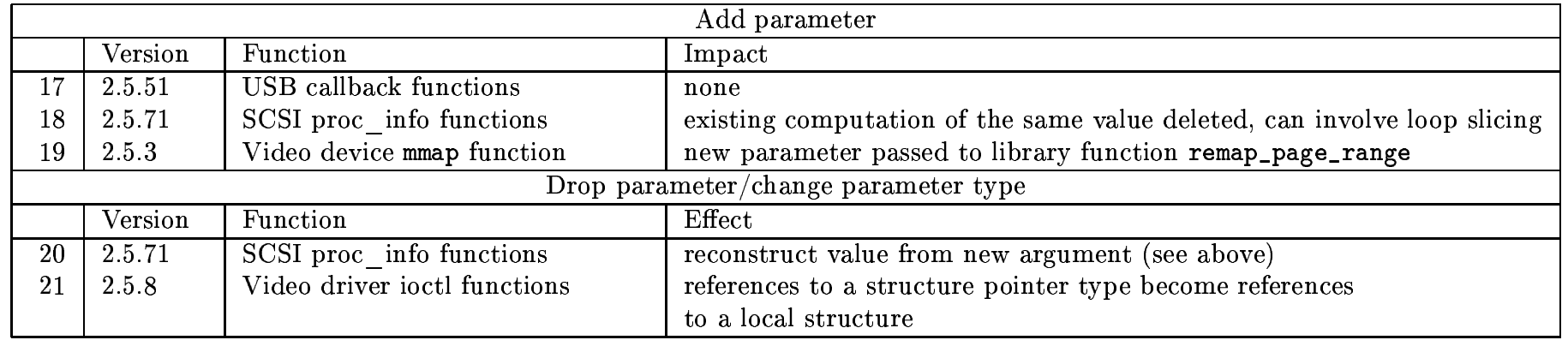

Data structures

\begin{tabular}{|l|l|l|l|}
\hline & Version & Structure type & Evolution \\
\hline 22 & 2.5 .45 & IsdnCardState, BCState & change field name \\
23 & 2.5 .27 & mddev_t & inline substructure \\
24 & 2.5 .67 & i2c_client & substructure introduced, getter/setter functions introduced \\
\hline
\end{tabular}

Protocols

Protocols
\begin{tabular}{|c|l|l|l|}
25 & 2.5 .52 & acpi_device_dir & insert assignment after call to remove_proc_entry in some contexts \\
26 & 2.5 .50 & irq_func callback function & drop parameter test, insert locking around function body \\
27 & 2.5 .36 & usb_stor_clear_halt & introduce error checking \\
28 & 2.5 .4 & network ioctl functions & $\begin{array}{l}\text { new ethtool cases, depending on whether the code defines } \\
\text { a debug variable }\end{array}$ \\
& & USB callback functions & interrupt urbs must now be explicitly resubmitted \\
29 & 2.5 .45 & conversion from i2c-old to i2c & many changes, including new functions to define, \\
30 & 2.5 .33 & & new structures to define and initialize, and new library functions to use \\
\end{tabular}

Figure 5: Collateral evolutions in Linux 2.5 
Collateral evolutions related to function return types again typically concern error return values. When a device-specific callback function that returns 0 or 1 is required to use more informative values, the collateral evolution entails identifying the existing values that indicate error or success, and choosing an appropriate value in each error case.

Data structures Evolutions in data structures typically involve adding, removing, or reorganizing fields, which trigger collateral evolutions similar to the changes in arguments and parameters described above. The collateral evolution may, however, be complicated by the use of local variables to name substructures, requiring a careful dataflow analysis to identify the affected code (line 23). In some cases, the reorganization of a structure is accompanied by the introduction of getter and setter functions, abstracting over the new access path (line 24). In the case of read accesses to the affected field, the collateral evolution may introduce a local variable to store the result of calling the getter function, rather than replacing every read access by a function call.

Protocols A driver support library protocol specifies the order in which various operations related to the functions and data structures exported by the library should be carried out. Such a protocol may for example specify a required sequence of function calls or a context in which error checking is needed. The instantiation of a protocol in device-specific code is often determined by the device-specific code structure. For example, when a protocol requires error checking, the actual code used to clean up in the case of an error may depend on the set of resources allocated by the device-specific code.

Protocol changes involve removing the instantiation of the old protocol from the device-specific code and inserting the appropriate instantiation of the new one. In some cases, the code to add or remove is fixed, and appears in a fixed context (line 25). In other cases, the instantiation of a protocol is context sensitive. For example, when locking is added, it must often be placed at every function return point; the positioning of function returns varies from function to function (line 26). When error checking is added, it may only be needed in code that does not already lead to an error return value (line 27). The set of new cases to be handled by an ioctl function may depend on the other features provided by the device-specific code (line 28). Finally, major reorganisations in an interface often involve a combination of these collateral evolutions (line 30).

\subsubsection{Assessment}

In some cases, the $\mathrm{C}$ compiler can help with collateral evolution, for example by detecting when a function is passed the wrong number of arguments. Nevertheless, the compiler only helps in cases where the need for the collateral evolution manifests itself as a type error; when $0 / 1$ return values are converted to error codes or the required sequencing of a set of function calls changes, the compiler provides no assistance.

The simplest collateral evolutions, such as renaming a function or adding a constant first argument, can be easily implemented using editor macros or shell scripts. There is indeed evidence that collateral evolution is done this way, as sometimes comments that coincidentally contain the name of an affected function are changed as well. Nevertheless, even in simple cases this approach is highly error prone, as it may modify code fragments that are unrelated to the collateral evolution, such as a function call where the name coincidentally contains the same text. More complex collateral evolutions require parsing complex expressions, analyzing the context, transforming multiple lines of code, and translating variable names and code patterns to those used in the affected file. The minor variations, omissions, and errors that we have observed in this process suggest that collateral evolution is often done by hand, which is time-consuming and error-prone.

\section{Case Studies}

In this section, we consider three collateral evolutions in detail. We have chosen these examples because they illustrate some of the more complex cases in three common categories: an argument added to a library function, a change in the required parameter type of a callback function, and a change in a protocol. We consider not only the modifications that the maintainer of device-specific code must make for each collateral evolution, but also the history of the collateral evolution, including a study of the bugs that were introduced.

\subsection{Addition of an argument}

The USB library function usb_submit_urb implements the passing of a message, implemented as USB Request Block (urb). This function uses the kernel memoryallocation function, kmalloc, which must be passed a flag indicating the circumstances in which blocking is allowed. Up through Linux 2.5.3, the flag was chosen in the implementation of usb_submit_urb as follows:

in_interrupt () ? GFP_ATOMIC : GFP_KERNEL

Comments in the file usb/hcd.c, however, indicate that this solution is unsatisfactory:

// FIXME paging/swapping requests over USB should not // use GFP_KERNEL and might even need to use GFP_NOIO ...

// that flag actually needs to be passed from the higher level.

Starting in Linux 2.5.4, usb_submit_urb takes one of the following as an extra argument: GFP_KERNEL (no 


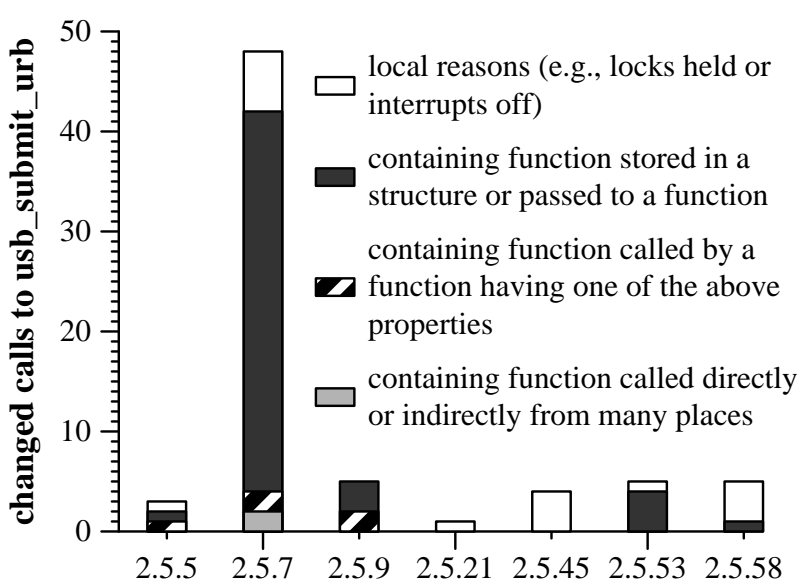

Figure 6: Linux 2.5 versions in which GFP_KERNEL is corrected to GFP_ATOMIC in a call to usb_submit_urb

constraints), GFP_ATOMIC (blocking not allowed), or GFP_NOIO (blocking allowed but not I/O). The programmer of device-specific code selects one of these constants according to the context of the call to usb_submit_urb.

Choosing the extra argument of usb_submit_urb requires a careful analysis of the surrounding code as well as an understanding of how this code is used by driver support libraries. Comments describing the relevant conditions are provided with the definition of usb_submit_urb starting in Linux 2.5.4. These comments state that GFP_ATOMIC is required in a completion handler, in code related to handling an interrupt, when a lock is held (including the lock taken when turning off interrupts), when the state of the running process indicates that the process may block, in certain kinds of network driver functions, and in SCSI driver queuecommand functions. Many of these situations, however, are not explicitly indicated by the code surrounding the call to usb_submit_urb. Instead, they require an understanding of the contexts in which the function containing the call to usb_submit_urb may be applied. In practice, this function can be passed to a driver support library via a data structure or function call and used in arbitrary ways, or can be invoked directly or indirectly by a local function that has one of the above properties.

The difficulty in understanding the conditions in which GFP_ATOMIC is required and identifying these conditions in driver code is illustrated by the many calls to usb_submit_urb that were initially transformed incorrectly. Figure 6 lists the versions in Linux 2.5 in which corrections in the use of usb_submit_urb occur and the reason for each correction. In each case, the error was introduced in Linux 2.5.4 or when the driver entered the kernel source tree, whichever came later. A major source of errors is the case where the function containing the call to usb_submit_urb is stored in a structure or passed to a function, as these cases require extra knowledge about how the structure is used or how the function uses its arguments. Indeed, in the serial subdirectory, all of the calls requiring GFP_ATOMIC fit this pattern and all were initially modified incorrectly (and corrected in Linux 2.5.7). Surprisingly, in 17 out of the 71 errors, the reason for using GFP_ATOMIC is locally apparent, reflecting either carelessness or insufficient understanding of the conditions in which GFP_ATOMIC is required. Indeed, in Linux 2.6.13, in the file usb/class/audio.c, GFP_KERNEL is still used in one function where interrupts are turned off.

The difficulty of choosing the value of the extra argument for usb_submit_urb is illustrated by the case of the function rt18150_start_xmit shown in Figure 2 The rtl8150 driver was introduced into the Linux source tree in Linux 2.5.8, at which point the call to usb_submit_urb in this function was given the argument GFP_KERNEL. This choice of argument is, however, incorrect, as rt18150_start_xmit is one of the kinds of network functions that requires GFP_ATOMIC. The code was corrected in Linux 2.5.9.

\subsection{Change in the type of a parameter}

A Linux ioctl function allows user-level interaction with a device driver. Copying arguments to and from user space is a tedious but essential part of the implementation of such a function. In Linux 2.5.7, the media support library introduced a wrapper function to encapsulate this argument copying. This function was refined in Linux 2.5.8 and named video_usercopy. As of Linux 2.6.13, video_usercopy was used in 31 media files and 6 usb files.

Introducing the use of video_usercopy affects the type of one of the parameters of the ioctl code. In the original version, this parameter is a pointer to user space, and each ioctl command must use the functions copy_from_user and copy_to_user to access or update its value. In these cases, the data is typically copied once, and otherwise accessed via a local data structure whose type is specific to the ioctl command. After the introduction of video_usercopy, the parameter of the ioctl function becomes a generic pointer to kernel space, which the ioctl code can read from or write to directly. The collateral evolution thus entails modifying the treatment of each ioctl command to remove the copy functions, casting the generic pointer parameter to a pointer of the structure type used by the command, and replacing the references to the local structure by pointer dereferences. The latter transformation can be quite invasive. For example, in the ioctl function of media/radio/radio-typhoon. c, $61 \%$ of the lines of code changes between Linux 2.5.6 and 2.5.8. 


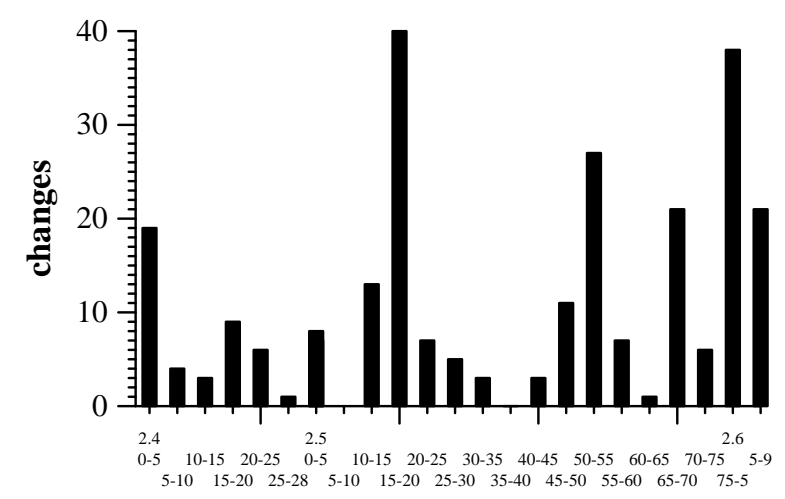

Figure 7: check_region elimination in Linux 2.4 to Linux 2.6

The behavior of video_usercopy is not specific to media drivers, and thus there has been interest in making the function more generally available [14]. Some evidence of the difficulties this may cause are provided by the case of i2c/other/tea575x-tuner.c in which video_usercopy was introduced in Linux 2.6.3. In this file, the calls to copy_from_user and copy_to_user were not removed. The bug was never fixed. Instead, the use of video_usercopy was removed from this file in Linux 2.6.8.

\subsection{Change in a function protocol}

The function check_region is used in the initialization of device drivers, in determining whether a given device is installed. In early versions of Linux, the kernel initializes device drivers sequentially [22]. In this case, a driver determines whether its device is attached to a given port using the following protocol: (i) call check_region to find out whether the memory region associated with the port is already allocated to another driver, (ii) if not, then perform some driver-specific tests to identify the device attached to the port, and (iii) if the desired device is found, then call request_region to reserve the memory region for the current driver. In more recent versions of Linux, the kernel initializes device drivers concurrently [6]. In this case, between the call to check_region and the call to request_region some other driver may claim the same memory region and initialize the device. To solve this problem, starting with Linux 2.4.2, device-specific code began to be rewritten to replace the call to check region in step (i) with a call to request_region, to actually reserve the memory region. Given this change, if in step (ii) the expected device is not found, then release_region must be used to release the memory region.

Eliminating a call to check_region requires replacing it by the associated call to request_region and inserting calls to release_region along error paths. In the first step, it is necessary to find the call to request_region that is associated with the given call to check_region. In practice, these are often not in the same function, requiring an interprocedural analysis. In the second step, it is necessary to identify code points at which it is known that the expected device has not been found and thus release_region is required. This condition is often indicated by the returning of an error value, but may also be indicated by going around a loop that checks successive ports until finding one with the desired device. At such code points, it may be the case that only a subset of the incoming paths contain a call to check_region. In these cases, the call to release_region must be placed under a conditional.

The elimination of check_region has been a recurring topic in Linux mailing lists, including the following exchange:

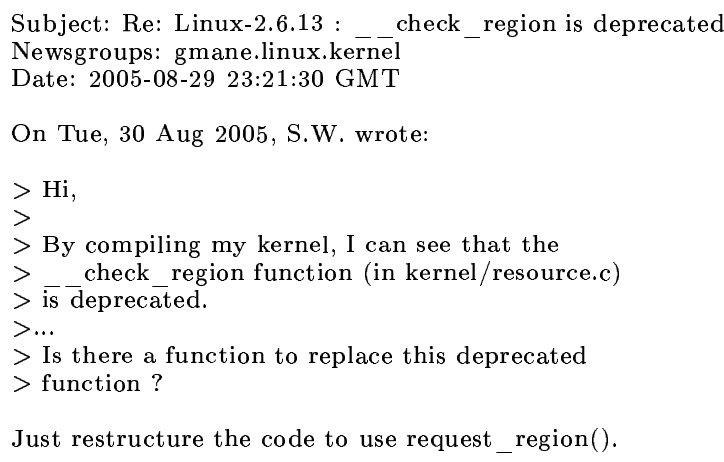

The response in this case does not convey any of the complexity of the collateral evolution process, and highlights the need for a formal language for specifying collateral evolutions. Indeed, both steps in eliminating check_region are difficult and time-consuming. This difficulty has lead to the slow pace of the evolution, as shown in Figure 7 Although beginning in Linux 2.4.2, released in February 2001, the evolution is still not complete as of Linux 2.6.13.3, released in October 2005.

\section{Quantitative Assessment}

In this section, we present a quantitative assessment of factors related to collateral evolution in Linux. We begin by assessing the complexity of the interdependencies of driver code based on the relationship between interfaces and device-specific files. We then consider the effect of evolution in the kernel and driver support libraries on interfaces. Finally, we quantify the required collateral evolutions. 


\subsection{Code base}

Our assessment is based on Linux code from version 2.2.0, released in January 1999, to version 2.6.13, released in August 2005. This sample contains both stable versions $(2.2,2.4$, and 2.6) and unstable ones (2.3 and 2.5). All files were obtained from http://www.kernel.org. Since 2.6.11, Linux been released in a series of subminor versions $(2.6 .11 .1,2.6 .11 .2$, etc.), which we do not consider separately. We focus on the drivers and sound directories. The sound directory is included because it was part of the drivers directory until Linux 2.5.5.

Our study distinguishes between driver support libraries and device-specific code. As there is no convention in Linux for identifying driver support libraries, we use the following heuristic. We consider that there is at most one driver support library per directory. A file is in the driver support library if it exports functions to multiple files or if it exports functions to another file in the driver support library. Other files are considered to be device-specific. We ignore both libraries that only export functions to other libraries and files that do not import any library functions, as these are not affected by the interface between driver support libraries and device-specific code that is the source of collateral evolution. We apply this algorithm to the files in the drivers, sound, and net directories. The net directory in included as a source of driver support libraries because network device drivers typically use its code. The kernel is considered to be another driver support library, defining all of the functions for which definitions are not found in the drivers, sound, or net code. In Linux 2.4.0 there are 66 driver support libraries and 874 device-specific files, while in the most recent version of Linux, 2.6.13, these numbers have more than doubled to 164 and 1926, respectively.

\subsection{Methodology}

A key point in our quantitative study is to understand the changes in device-specific code from one version of Linux to the next. For this purpose, we have developed a tool that detects commonalities and differences in two versions of $\mathrm{C}$ code. The tool starts from a patch file, in which it analyzes each of the identified difference regions. We illustrate the analysis using the difference region shown in Figure 8 that is derived from a patch file comparing the definition of the function rt18160_start_xmit when it was introduced in Linux 2.5.8 to the most recent version in Linux 2.6.13. The complete Linux 2.6.13 definition of rt18160_start_xmit was previously shown in Figure 2

The first step in the analysis is to align the common parts of the two fragments and to identify the maximally different regions. In our example, the maximally different regions are as follows:

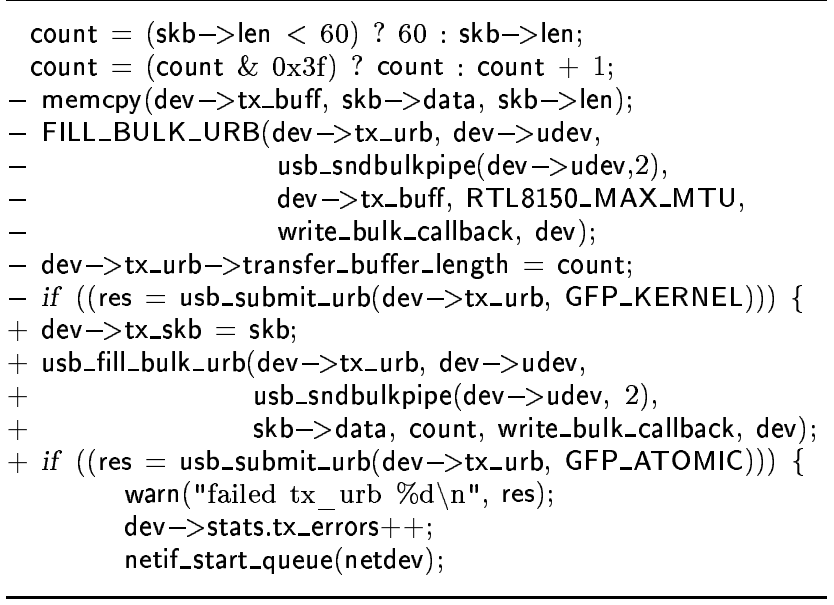

Figure 8: Extracts of a patch derived from the rtl8150 driver

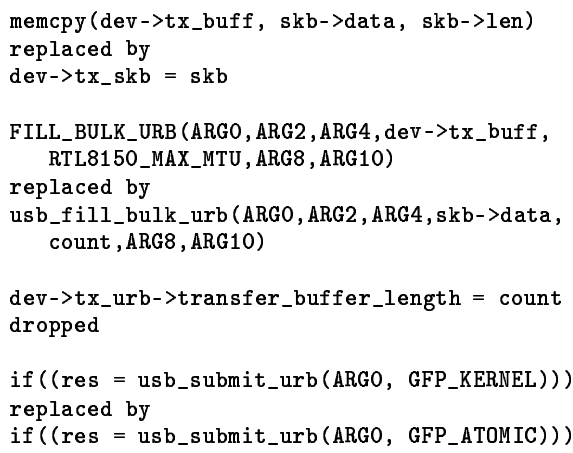

In this result, the various statements of the dropped and added regions are matched up line by line except for the second assignment. This assignment is considered by itself because the next element in both fragments is a conditional test, and these are aligned instead. We next observe that when a function call is matched with another function call, the common arguments are replaced by a term ARG $n$, where $n$ is determined by the argument position. As the common arguments are not possible evolutions, we perform this normalization to improve the chance that this pair of calls will match with other calls to the same functions. Finally, we observe that the calls to usb_submit_urb have a non-trivial common context, including an assignment and a conditional test. This occurs because conditionals, assignments and function calls that have a top-level difference among their subterms are considered to be different as well.

The next step is to distinguish between differences that are specific to a single device-specific file and differences that are recurrent across a Linux version, and thus represent a collateral evolution. For this, we use a threshold: a difference is considered to be part of a collateral evolution if it occurs at least 5 times and these occurrences are distributed across at least 3 files. It may, however, be the case that a complete maxi- 
mally different region does not occur often enough to satisfy the threshold, but there is some subterm that represents the collateral evolution. An example is the case of the conditional test identified in the case of rt18160_start_xmit:

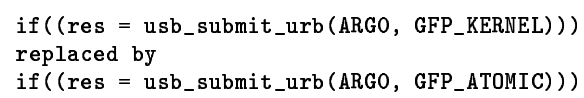

We have previously identified the correction of the second argument to usb_submit_urb as part of a collateral evolution, but it may not be the case that every use of usb_submit_urb occurs nested in the assignment and conditional test shown here. For each maximal difference, the analysis constructs a tree of the term, its subterms, and abstractions of the subterms, in which e.g. function arguments are replaced by an arbitrary value CODE. The analysis then works downwards from the root of the tree to find the maximal terms that match with enough other differences to satisfy the threshold. In the version where the correction to the argument of usb_submit_urb appears, the matching process reaches the call to usb_submit_urb itself, because in this version the other updates to calls to usb_submit_urb occur in varying contexts.

We use this analysis not only to detect collateral evolution sites, as described in Section [5.5. but also to detect evolutions in interfaces themselves, as described in Section 5.4. Specifically, a collateral evolution that directly affects an interface element is also an indicator of an evolution in the interface. We follow this strategy for detecting interface changes, rather than e.g., looking for changes in header files, because it can detect changes related to values and protocols, while header files only indicate type changes.

\subsection{Interfaces}

Because collateral evolution is derived from interface changes, the size and distribution of interfaces is a measure of the potential difficulty of collateral evolution in device-specific code. We consider the relationship between interfaces and device-specific code from the perspective of the maintainer of a single device-specific file and from the perspective of the library developer.

Interface complexity from the perspective of the maintainer of device-specific code The number of library functions used by device-specific code is a measure of its complexity, as the maintainer must understand each of these functions, including its arguments and associated protocols. Figure 9 a shows the number of library functions used by each device-specific source file. This figure shows clearly that not only has the size of the driver code doubled in the last five years since Linux 2.4.0, but also the complexity, with substantially more device-specific files referring to up to 20 library functions in Linux 2.6.13 than in Linux 2.4.0.
Furthermore, in Linux 2.4.0 the largest number of library function references per file is 36 while in Linux 2.6.13 this number has jumped to 59 .

We may further refine the assessment of the complexity of device-specific code by taking into account the library structure. Each driver support library represents a unit of understanding, and thus code that relies on multiple driver support libraries requires more expertise to maintain than code that relies on only one. Figure 9b also shows the number of libraries on which each file depends. In Linux 2.4 .0 only 169 files rely on three or more libraries, while in Linux 2.6.13 this number has increased to 501.

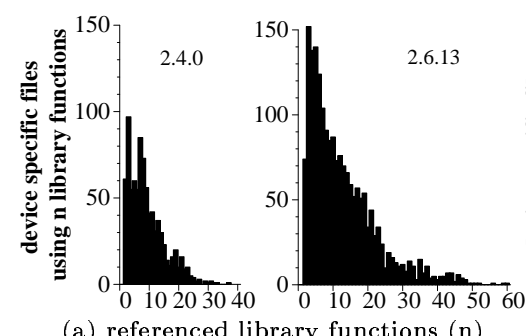

(a) referenced library functions (n) per device-specific file

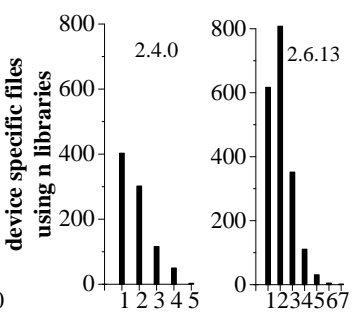

(b) referenced libraries (n) per device-specific file
Figure 9: (a) Library function references and (b) library references in Linux 2.4.0 and Linux 2.6.13

Interface complexity from the perspective of the developer of a driver-support library We measure the complexity of an interface in terms of the number of functions it exports, as shown in Figure [10. While the number of functions exported by the typical interface is under 20 in both Linux 2.4.0 and Linux 2.6.13, the maximum number of exported functions increases significantly, from around 80 in Linux 2.4.0 to around 130 in Linux 2.6.13.

When a change occurs in the interface of a driver support library, collateral evolution is needed in all dependent device-specific code. The difficulty of performing this collateral evolution depends not only on the number of files involved, but also on the distribution of these files across different directories, as files in other directories may not be known to the library developer and may exhibit unique code patterns. Figures 10b and 10. show the number of device-specific files depending on each interface and the number of directories containing at least one device-specific file with such a dependency. Again, the maximum number has doubled between Linux 2.4.0 and Linux 2.6.13. In Linux 2.6.13, the most widely used library is PCI, which is the basic bus used on PCs. The network device and ethernet support libraries are the next most widely used. The USB support library is also among the most widely used, being used by 131 files. 


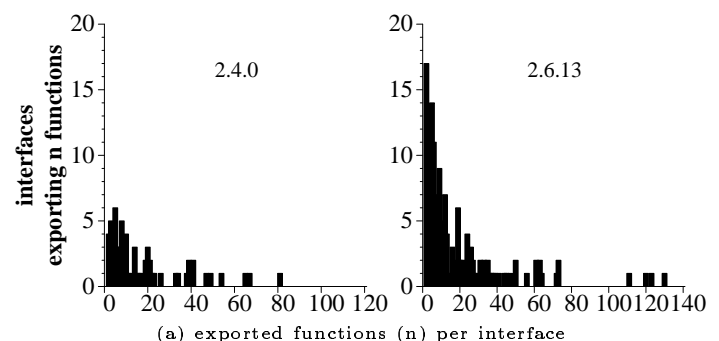

(a) exported functions (n) per interface

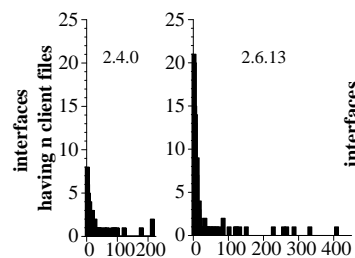

(b) client files (n) per interface

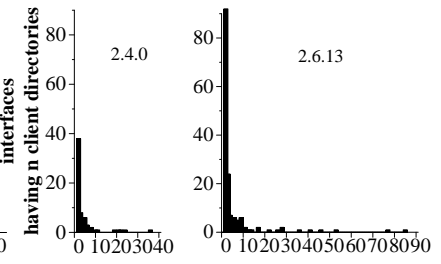

(c) client directories (n) per interface
Figure 10: (a) Interface size and (b,c) interface usage in Linux 2.4.0 and Linux 2.6.13

\subsection{Quantitative assessment of evolu- tion}

Figure 11] shows the number of evolutions that have occurred in library functions, device-specific callback functions, data structures, and protocols in the versions of Linux between 2.2 and 2.6. In the current state of our tool, protocols are detected only as the addition or deletion of single function calls. The most significant number of evolutions occurs in library functions and protocols, with an increase across the versions of Linux that roughly mirrors the increase in code size.

It is interesting to compare the number of evolutions in the unstable versions 2.3 and 2.5 and their stable derivatives 2.4 and 2.6. The stable Linux 2.4 had slightly more evolutions than the unstable version 2.3 , but was the main version of Linux for almost three years, while Linux 2.3 was only under development for 1 year. In the case of Linux 2.5 and Linux 2.6, the so-called stable Linux 2.6 has had almost as many evolutions as the unstable Linux 2.5. The current age of Linux 2.6 is about the same as the time in which Linux 2.5 was under development, but we can expect Linux 2.6 to be the main version for some time longer, and thus to accumulate even more evolutions.

\subsection{Quantitative assessment of collat- eral evolution}

Figure 12 assesses the number of lines modified due to collateral evolutions from the first patch file for Linux 2.2 to the patch file for Linux 2.6.13. We observe that while the number of lines affected by collateral evolutions varies from one version to the next, there is a general increasing trend, with a significant increase in

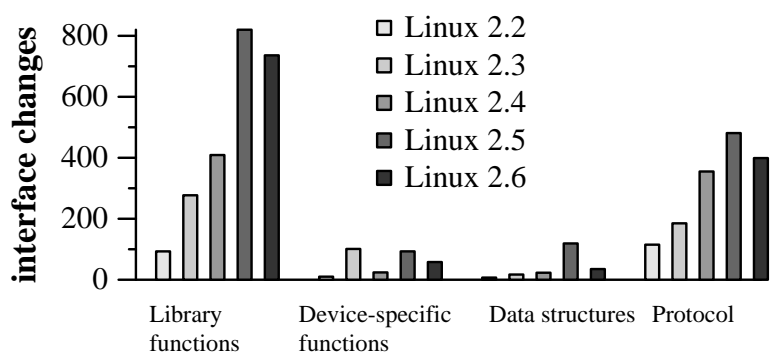

Figure 11: Number of evolutions in interface elements in Linux 2.2 to Linux 2.6

Linux 2.6. In terms of the percentage of lines modified due to collateral evolution as compared to the the number of lines modified overall in device-specific code, we see that the biggest spikes, of over $35 \%$, occur in the unstable versions. We conjecture that OS developers postpone evolutions that may induce many collateral evolutions until these versions, due to the amount of work that they entail.
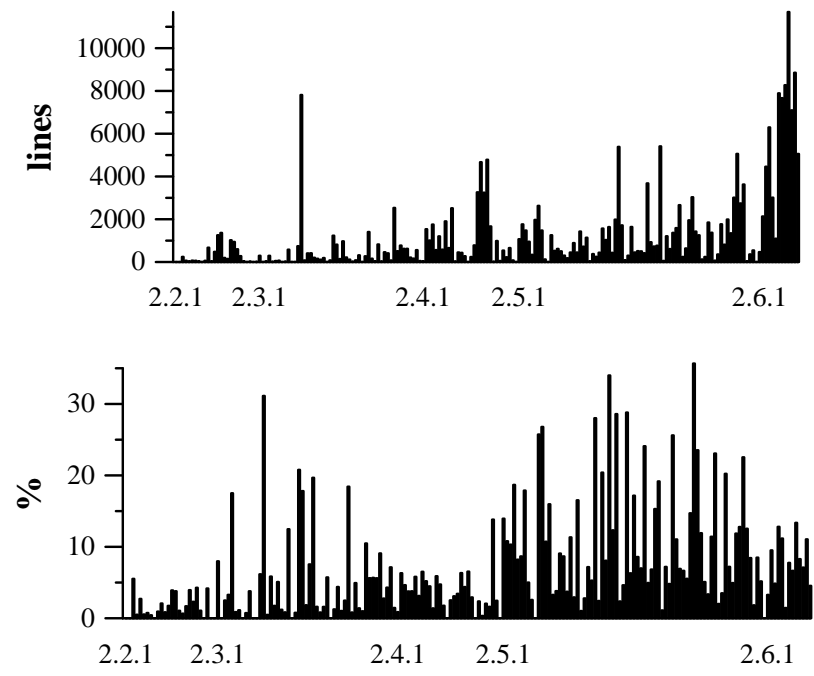

Figure 12: Patch file lines and percentage of patch file lines derived from device-specific code and containing collateral evolutions in Linux 2.2 to Linux 2.6

Figure 13 measures the magnitude of individual collateral evolutions in terms of the number of sites affected and terms of the number of files affected. On average, a collateral evolution is required at around 14 sites and in a total of 10 files. Nevertheless, many collateral evolutions are much more pervasive, with one change in library function affecting around 1000 sites in Linux 2.6. Overall, collateral evolutions in library functions and protocols affect both the most sites and files. Collateral evolutions in library functions vary from simple textual replacement to cases that involve 
careful analysis of the source code. Protocol changes that involve adding new functions, on the other hand, are often difficult, as they require situating new code within a context whose precise structure can vary.
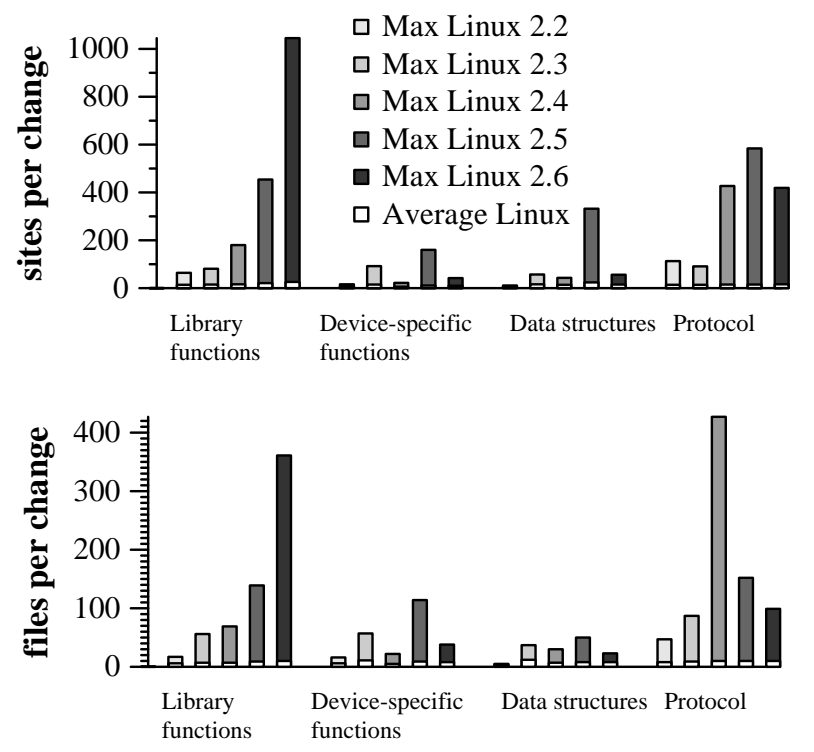

Figure 13: Maximum number of sites and files affected by a single collateral evolution

\section{Requirements for Coccinelle}

Ultimately, our goal is to develop the Coccinelle tool providing automated assistance to ease the collateral evolution problem. This assistance will comprise a transformation language for describing collateral evolutions and a transformation engine for applying them to device-specific code. We envision that Coccinelle will be used as follows. When a developer modifies the interface of a driver support library, he also uses the transformation language to specify the associated collateral evolution. He then uses the transformation engine to apply the specified collateral evolution to device-specific code in the Linux source tree. When the specification has been validated on the available sources, the developer makes it publicly available for use by the maintainers of drivers outside the Linux source tree.

In this section, we use the results of our analysis of collateral evolutions in Linux device-specific code to suggest a number of features that Coccinelle should provide.

\subsection{Support for C code}

$\mathrm{C}$ code is notoriously difficult to process automatically, for reasons including the size of the language, the am- biguity between type names and function names, and the possibility of arbitrary code reorganizations using macros. A typical solution is to apply the $\mathrm{C}$ preprocessor to the code, and then to rewrite the code into a simplified intermediate representation [5, 21. The result is then expressed in this simpler sublanguage.

Simplifying the source code, however, is not appropriate for implementing collateral evolution. Collateral evolution is intended to create code that will continue to evolve and be maintained, and thus the result must be in the form of the original source code, including macro definitions, comments, and whitespace. Furthermore, collateral evolutions may involve macro calls (e.g. line 3 in Figure [5), which thus must be visible to the transformation engine. More generally, the transformation rule developer must have confidence that the rule will apply where he expects and will not apply where he does not expect, without needing to understand the simplification process. These issues thus imply that Coccinelle must provide facilities for processing and constructing code following the structure and conventions of the original source program.

\subsection{Mechanisms for specifying code fragments}

To specify transformation rules that are applicable to multiple device-specific files, it is necessary to identify relevant code fragments in a generic way. Devicespecific code can be written in many different styles, using varying naming and coding conventions. A point in common, however, is that collateral evolutions are triggered by changes in driver support library interfaces and that device-specific code must use these interfaces in a standard way. Thus, the transformation language should provide abstractions that identify code in terms of the interface elements identified in Section 3.1 calls to library functions, definitions of devicespecific callback functions, accesses to interface data structures and protocol relationships. These points become anchors from which to reason about code in the context affected by collateral evolution. For example, the collateral evolution of usb_submit_urb (Section 4.1) requires searching from the call site for the taking and releasing of locks in the context, while the case of video_usercopy (Section 4.2) requires first identifying the callback function whose prototype has changed and then reasoning about its local variables.

\subsection{Mechanisms for describing rela- tionships between code fragments}

All of the examples described in Section 4 and many of the examples summarized in Figure 5 depend in some way on control-flow and data-flow information. For example, in the case of check_region (Section 4.3), control-flow analysis is needed to make the connection 
between the call to check_region and the corresponding call to request_region in the source code, while in the case of usb_submit_urb (Section 4.1) data-flow analysis is needed to trace the use of the function calling usb_submit_urb when this function is stored in a pointer and invoked via an indirect function call. In many cases, these analyses must be inter-procedural.

The need for program analyses raises the issue of who should implement them and how to access their results. One approach is to allow developers to encode analyses within the transformation rules [24]. This approach allows the analyses to be finely tailored to the needs of the transformation, but can massively complicate the transformation rules. It thus seems necessary to build the analyses into the transformation engine. Program analyses in their full generality, however, are very time consuming, and our study shows that collateral evolutions are sufficiently localized that complete precise analysis of entire device-specific files is not needed. Thus, a mechanism is needed to limit the analyses to the needs of a given transformation rule. Finally, suitable abstractions must be provided in the transformation language for accessing the results of analyses. Lacey et al. have proposed a form of rewrite rule that specifies the original code, the transformed code, and a side-condition expressed using temporal logic that describes relationships between the original code and its context in a control-flow graph [16. We have used this approach in automating the reengineering of Linux for use with the scheduling framework Bossa [1, 20, and expect that, generalized to also allow specification of data-flow relationships, it will be useful in describing collateral evolutions as well.

\section{Conclusion}

In this paper, we have shown that the evolution of drivers is a critical issue. Nevertheless, this issue has received little attention from researchers. Our long term goal is to design Coccinelle, a tool that provides a means for expressing knowledge about collateral evolutions and a transformation engine to assist developers in applying them. As such, the domain analysis conducted in this work represents a first step towards making collateral evolution easy and robust, in order to improve the reliability of device support in operating systems.

Based on our these results, our next effort will be in the design of a formal language for expressing collateral evolution and the associated transformation engine. As a proof of concept, we plan to return "back to the future" of Linux 2.4 and replay the evolution to Linux 2.6 .

\section{References}

[1] R. A. Åberg, J. L. Lawall, M. Südholt, G. Muller, and A.-F. Le Meur. On the automatic evolution of an OS kernel using temporal logic and AOP. In Proceedings of the 18th IEEE International Conference on Automated Software Engineering (ASE 2003), pages 196-204, Montreal, Canada, Oct. 2003. IEEE.

[2] J. Appavoo, M. Auslander, M. Burtico, D. D. Silva, O. Krieger, M. Mergen, M. Ostrowski, B. Rosenburg, R. W. Wisniewski, and J. Xenidis. K42: an open-source Linux-compatible scalable operating system kernel. IBM Systems Journal, 44(2):427-440, 2005.

[3] T. Ball and S. K. Rajamani. Automatically validating temporal safety properties of interfaces. In M. Dwyer, editor, 8th International SPIN Workshop, number 2057 in Lecture Notes in Computer Science, Toronto, Canada, 2001.

[4] A. Chou, J. Yang, B. Chelf, S. Hallem, and D. Engler. An empirical study of operating systems errors. In SOSP'01 [23], pages 73-88.

[5] C. Consel, J. L. Lawall, and A.-F. Le Meur. A tour of Tempo: a program specializer for the $\mathrm{C}$ language. Science of Computer Programming, 52(1$3): 341-370,2004$.

[6] A. C. de Melo, D. Jones, and J. Garzik, 2001. http://umeet.uninet.edu/umeet2001/talk/15-122001/ arnaldo-talk.html.

[7] D. R. Engler, B. Chelf, A. Chou, and S. Hallem. Checking system rules using system-specific, programmer-written compiler extensions. In Proceedings of the Fourth USENIX Symposium on Operating Systems Design and Implementation (OSDI), pages 1-16, San Diego, CA, Oct. 2000.

[8] D. R. Engler, D. Y. Chen, A. Chou, and B. Chelf. Bugs as deviant behavior: A general approach to inferring errors in systems code. In SOSP'01 [23], pages $57-72$.

[9] J.-P. Fassino, J.-B. Stefani, J. Lawall, and G. Muller. THINk: A software framework for component-based operating system kernels. In 2000 USENIX Annual Technical Conference, pages 73-86, Monterey, CA, June 2002.

[10] B. Ford, G. Back, G. Benson, J. Lepreau, A. Lin, and O. Shivers. The Flux OSKit: A substrate for kernel and language research. In Proceedings of the 16th ACM Symposium on Operating Systems Principles (SOSP'97), pages 38-51, Saint-Malo, France, Oct. 1997. 
[11] J. S. Foster, T. Terauchi, and A. Aiken. Flowsensitive type qualifiers. In Proceedings of the 2002 ACM SIGPLAN conference on Programming Language Design and Implementation, pages 38-51, Berlin, Germany, June 2002.

[12] M. Fowler, K. Beck, J. Brant, W. Opdyke, and D. Roberts. Refactoring: Improving the Design of Existing Code. Addison-Wesley Professional, 1999.

[13] A. E. Hassan. Mining Software Repositories to Assist Developers and Support Managers. PhD thesis, School of Computer Science, Faculty of Mathematics, University of Waterloo, Ontario, Canada, 2004.

[14] C. Hellwig, http://www.cs.helsinki.fi/linux/linux2003. kernel/2003-20/1120.html.

[15] P. Koellner, $\quad$ Feb. 2002. http://www.uwsg.iu.edu/hypermail/ linux/kernel/0202.2/0106.html.

[16] D. Lacey and O. de Moor. Imperative program transformation by rewriting. In R. Wilhelm, editor, Compiler Construction, 10th International Conference, CC 2001, number 2027 in Lecture Notes in Computer Science, pages 52-68, Genova, Italy, 2001.

[17] Z. Li, S. Lu, S. Myagmar, and Y. Zhou. CP-Miner: A tool for finding copy-paste and related bugs in operating system code. In Proceedings of the Sixth USENIX Symposium on Operating Systems Design and Implementation (OSDI), pages 289-302, San Fransisco, CA, Dec. 2004.

[18] LWN. API changes in the 2.6 kernel series, Oct. 2005. http://lwn.net/Articles/2.6-kernel-api/.

[19] D. S. Miller, $\quad$ Feb. 2002. http://www.ussg.iu.edu/hypermail/ linux/kernel/0202.1/0855.html.

[20] G. Muller, J. Lawall, J.-M. Menaud, and M. Südholt. Constructing component-based extension interfaces in legacy systems code. In ACM SIGOPS European Workshop 2004 (EW2004), pages 80-85, Leuven, Belgium, Sept. 2004.

[21] G. C. Necula, S. McPeak, S. P. Rahul, and W. Weimer. CIL: Intermediate language and tools for analysis and transformation of $\mathrm{C}$ programs. In R. N. Horspool, editor, Compiler Construction, 11th International Conference, CC 2002, number 2304 in Lecture Notes in Computer Science, pages 213-228, Grenoble, France, Apr. 2002.

[22] A. Rubini and J. Corbet. Linux Device Drivers, 2nd Edition. O'Reilly, June 2001.
[23] Proceedings of the 18th ACM Symposium on Operating System Principles, Banff, Canada, Oct. 2001.

[24] E. Visser. Program transformation with Stratego/XT: Rules, strategies, tools, and systems in StrategoXT-0.9. In C. Lengauer et al., editor, Domain-Specific Program Generation, number 3016 in Lecture Notes in Computer Science, pages 216-238, 2004.

[25] D. Wambolt, 2001. http://seclists.org/lists/linuxkernel/2001/Dec/2027.html.

[26] J. Weber, Feb. 2002. http://www.ussg.iu.edu/hypermail /linux/kernel/0202.1/0697.html. 


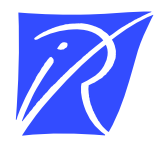

Unité de recherche INRIA Rennes

IRISA, Campus universitaire de Beaulieu - 35042 Rennes Cedex (France)

Unité de recherche INRIA Futurs : Parc Club Orsay Université - ZAC des Vignes

4, rue Jacques Monod - 91893 ORSAY Cedex (France)

Unité de recherche INRIA Lorraine : LORIA, Technopôle de Nancy-Brabois - Campus scientifique

615, rue du Jardin Botanique - BP 101 - 54602 Villers-lès-Nancy Cedex (France)

Unité de recherche INRIA Rhône-Alpes : 655, avenue de l'Europe - 38334 Montbonnot Saint-Ismier (France)

Unité de recherche INRIA Rocquencourt : Domaine de Voluceau - Rocquencourt - BP 105 - 78153 Le Chesnay Cedex (France)

Unité de recherche INRIA Sophia Antipolis : 2004, route des Lucioles - BP 93 - 06902 Sophia Antipolis Cedex (France)

INRIA - Domaine de Voluceau - Rocquencourt, BP 105 - 78153 Le Chesnay Cedex (France)

http://www.inria.fr

ISSN 0249-6399 